\title{
Miszelle
}

Eugenio Villa*

\section{Sull'unità dei trattati de barbarismo, de soloecismo e de acyrologia di Polibio di Sardi}

https://doi.org/10.1515/phil-2020-0131

Keywords: Polibio di Sardi, acirologia, critica testuale, grammatica antica

Una decina di manoscritti vergati tra la metà del XIII e l'inizio del XVI secolo riporta cinque brevi opuscoli grammaticali attribuiti a un certo Polibio di Sardi: ${ }^{1}$

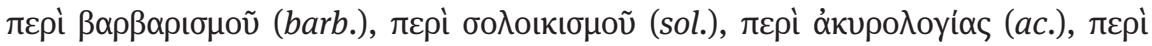

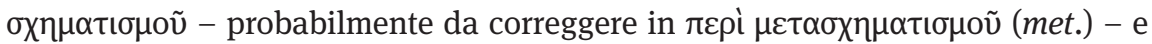

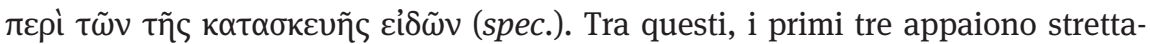
mente connessi tra di loro in quanto, a introdurre la trattazione sul barbarismo, troviamo una definizione per gradi degli "errori relativi alle parole" che individua per l'appunto barbarismo, solecismo e acirologia:

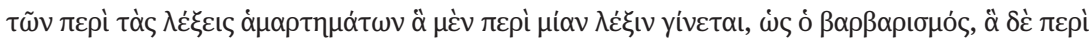

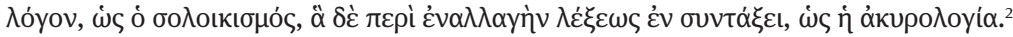

1 Una fragile ricostruzione su chi potesse essere questo grammatikós si trova in Ch. Jones, "Polybius of Sardis”, Classical Philology 91, 1996, 247-253. Per i manoscritti utilizzo le sigle stabilite da M. G. Sandri, Trattati greci su barbarismo e solecismo. Introduzione ed edizione critica (Untersuchungen zur antiken Literatur und Geschichte 135), Berlin 2020. In particolare, alla famiglia $\boldsymbol{\alpha}$ appartengono i codici Città del Vaticano, Biblioteca Apostolica Vaticana, Urb. gr. 152 (V), Oxford, Bodleian Library, Bar. 216 (x), Paris, Bibliothèque nationale de France, gr. 2552 (e), Salamanca, Biblioteca Universitaria, 242 (S) e alla famiglia $\boldsymbol{\beta}$ i codici Firenze, Biblioteca Medicea Laurenziana, Plut. 87.10 (F), Madrid, Biblioteca nacional de España, gr. 4576 (L), Madrid, Biblioteca nacional de España, gr. 7211 (N), Praga, Národní knihovna České republiky, XXV C 31 (Q), Venezia, Biblioteca Nazionale Marciana, gr. X 26 coll. 1305 (M).

2 Le più recenti edizioni critiche degli opuscoli polibiani sono contenute in Sandri (n. 1) 237-244 e E. Villa, Polybii Sardiani: De barbarismo, soloecismo et acyrologia. Un esperimento di edizione critica, Tesi di Laurea, Udine 2016.

*Indirizzo di corrispondenza: Eugenio Villa, Scuola Normale Superiore, Classe di Lettere e Filosofia, Piazza dei Cavalieri 7, Pisa, IT-56126, E-Mail: eugenio.villa@sns.it 
Seppur in maniera latente, anche la recensio sembra confermare questo legame; infatti, delle due famiglie che trasmettono gli opuscoli polibiani, i testimoni della prima $(\boldsymbol{\alpha})$ riportano barb. seguito da sol. e quelli della seconda ( $\boldsymbol{\beta})$ riportano sol. seguito da $a c$. (e met. e spec.), ai quali va poi aggiunto il Paris, Bibliothèque nationale de France, supplement grec $58(\mathbf{R})^{3}$ che riporta soltanto ac. e l'inizio di met. Sol. svolge quindi la funzione di trait d'union tra i capostipiti $\boldsymbol{\alpha}$ e $\boldsymbol{\beta}$, suggerendo che l'Ausgangstext conteneva barb. seguito da sol. e ac., o come tre sezioni di uno stesso opuscolo grammaticale o come tre opuscoli comunque da leggere assieme. La divisione dei tre testi nel corso della tradizione andrà attribuita alla scelta dei copisti: difficilmente una caduta materiale può comportare la perdita di una sezione lasciando le altre intere. ${ }^{4}$

Tuttavia, a causa del fatto che nessun manoscritto trasmette assieme i tre testi, essi non sono mai stati pubblicati in una stessa sede: nel 1759 Iriarte trascrisse sol. e ac. da $\mathbf{L}$ nella relativa scheda catalografica, segnando poi le varianti di $\mathbf{N}$ in quella dedicata a quest'ultimo, ${ }^{5}$ nel 1831 Boissonade pubblicò barb. e sol. a partire da e (il quale è l'unico della classe $\boldsymbol{\alpha}$ a riportare indicazione circa titolo e autore), ${ }^{6}$ nel 1833 Cramer pubblicò barb. e sol. a partire da $\mathbf{x}^{7}$ e nel 1867 Nauck curò la prima edizione critica di barb. e sol. a partire dalle edizioni di Boissonade e Cramer. ${ }^{8}$ In particolare, Boissonade, Cramer e Nauck, disponendo solo di testimoni del ramo $\boldsymbol{\alpha}$, ritenevano che barb. e sol. fossero le uniche due sezioni di una stessa opera che Boissonade separa inoltre esplicitamente da $a c .{ }^{9}$ Questa stessa distinzione si trova poi nella voce dedicata a Polibio di Sardi dalla $R E^{10}$ ed è sopravvissuta anche nella recente edizione critica curata da Sandri, la

3 Descrizione in C. Astruc/C. Belon/M.-L. Concasty/Ch. Förstel, Catalogue des manuscrits grecs. Supplément grec numéros 1 à 150, Paris 2003, 209-210. Il manoscritto è assente nello studio di Sandri perché non contiene barb. e sol.

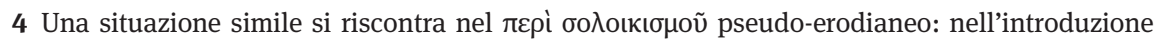
vengono distinti solecismo, barbarismo e acirologia, i quali dovevano essere tutti presenti nell' $\mathrm{Au}$ sgangstext, ma "a un certo punto della trasmissione del trattato pseudo-erodianeo sono andate smarrite le sezioni relative al barbarismo e all'akyrologhía" poi colmate con altri testi nel subarchetipo $\theta$; vd. Sandri (n. 1) 53.

5 Vd. J. de Iriarte, Regiae Bibliothecae Matritensis Codices Graeci Manuscripti, vol. 1, Madrid 1759, 148-149; 373.

6 Vd. Anecdota Graeca, vol. 3, ed. J. Fr. Boissonade, Paris 1831, 229-237.

7 Vd. "Anecdota Barocciana”, ed. I. A. Cramer, in: J. Ch. Hare (cur.), The Philological Museum, vol. 2, Cambridge 1833, 108-116.

8 Vd. Lexicon Vindobonense. Accedit Appendix duas Photii homilias et alia opuscola complectens, ed. A. Nauck, Sankt Petersburg 1867, 283-289.

9 Vd. Boissonade (n. 6) 229 n. 2: "Polybii ipsius exstat observatio de acyrologia”.

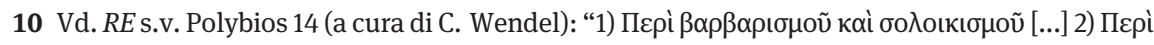

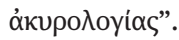


quale, nonostante circoscriva il suo studio ai trattati greci su barbarismo e solecismo - anche se uno dei suoi testi è inserito in una più ampia trattazione grammaticale, ella non la edita quindi per intero -, nondimeno in altri due casi mantiene la sezione dedicata all'acirologia dei testi che edita. ${ }^{11} \mathrm{E}$ si noti inoltre che, eccezion fatta per $\mathbf{Q}$, nella descrizione del contenuto di ciascun testimone del ramo $\boldsymbol{\beta}$ Sandri parla sempre del "trattato sul barbarismo e il solecismo", ${ }^{12}$ nonostante tali testimoni, come già visto, contengano soltanto la trattazione sul solecismo.

Ac. non viene quindi stampato da quasi tre secoli e la trascrizione di Iriarte, oltre a riprodurre tutti gli errori di $\mathbf{L}$, ne aggiunge di propri. Pertanto, offro qui di seguito l'edizione critica di $a c$. accompagnata da una traduzione e da alcune brevi note critico-testuali; essa deve comunque essere intesa e letta dopo l'edizione di barb. e sol. Per stabilire il testo ho collazionato tutti i testimoni noti, ossia $\mathbf{F}, \mathbf{N}$ (assieme ai suoi descripti $\mathbf{L}$ e $\mathbf{M}$ ), $\mathbf{Q}$ e R. Eccezion fatta per F, il quale omette la definizione di acirologia e la maggior parte degli esempi, gli altri testimoni presentano un testo abbastanza stabile, ma non mancano errori, riduzioni e ampliamenti, tutti registrati in apparato. In due casi sono intervenuto nel testo, ritenendo non soddisfacente la ricostruzione di $\boldsymbol{\beta}$ : a 1 . 1 ho integrato una negazione

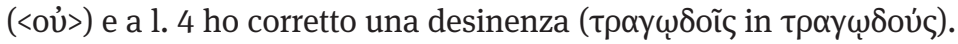

La punteggiatura dei manoscritti è sostanzialmente omogenea, soprattutto grazie al fatto che il testo è composto da una serie di esempi giustapposti. $\mathbf{N}$ e $\mathbf{M}$ utilizzano solo la teleía stigmé per separare gli elementi delle enumerazioni sia asindetiche sia polisindetiche, L utilizza la teleía stigmé per le enumerazioni asindetiche e il díkolon per le enumerazioni polisindetiche e F, $\mathbf{Q}$ e $\mathbf{R}$ utilizzano la virgola con valore rematico e la teleía stigmé per separare gli elementi delle enumerazioni sia asindetiche sia polisindetiche; tutti indicano la fine del testo con díkolon seguito da trattino. Per rispettare la punteggiatura dei testimoni, senza tuttavia scadere in usi eccessivamente anacronistici, utilizzo la virgola per le enumerazioni asindetiche, la teleía stigmé per le enumerazioni polisindetiche e il díkolon seguito da trattino per segnalare la fine del testo.

11 Vd. Sandri (n. 1) 21 n. 101, dove elenca i trattati polibiani: "Oltre a quello sul barbarismo e sole-

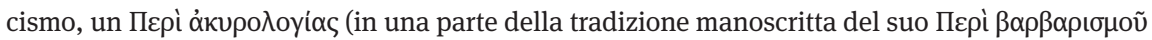

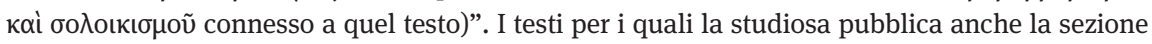
dedicata all'acirologia sono il [5] e il [12].

12 Vd. Sandri (n. 1) 87 [F]; 88 [L e N]; 93 [M]. 


\section{Testo critico}

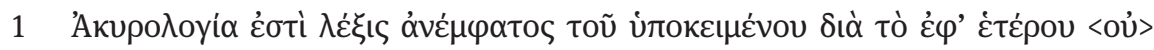

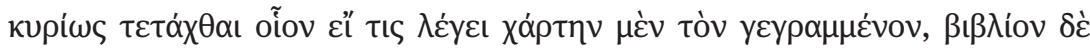

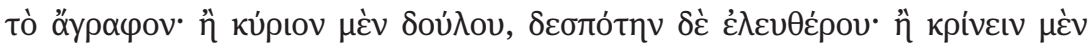

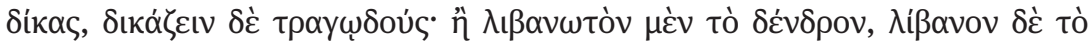

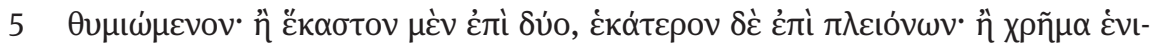

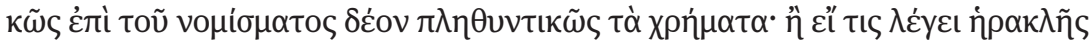

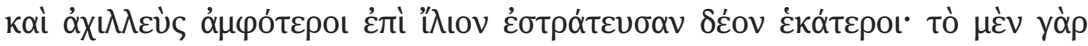

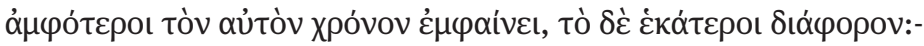

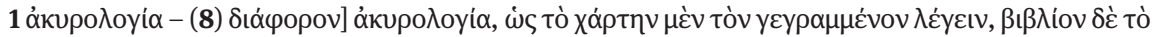

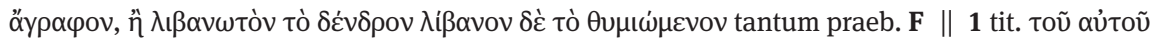

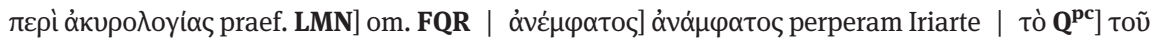

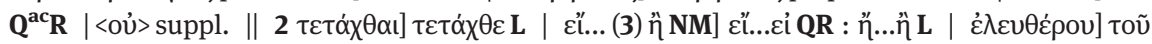

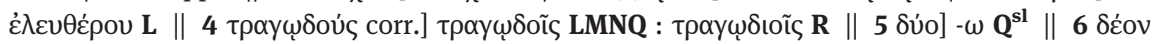

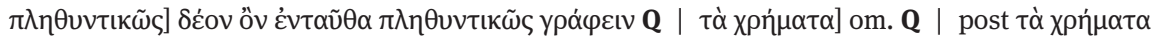

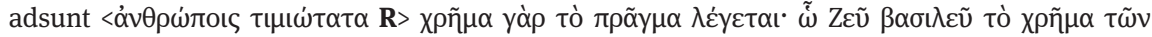

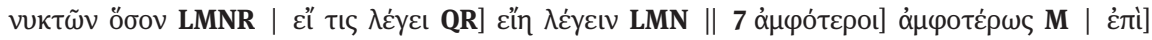

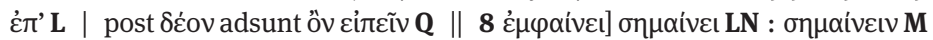

\section{Traduzione}

L'acirologia è un'espressione che non indica correttamente ciò di cui si parla a causa del suo esser stata impropriamente riferita a un'altra cosa, come se qualcu-

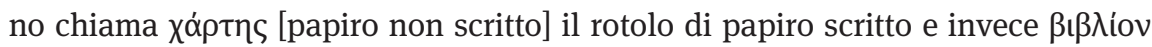
[libro] quello non scritto, oppure se dice kúpış [capo] in riferimento all'autorità

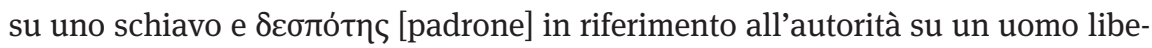

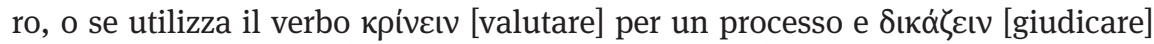
per i tragediografi, o se chiama $\lambda$ ı $\beta \alpha \omega \tau$ tós [incenso] l'albero e $\lambda i \beta \alpha v o \varsigma$ [albero dell'incenso] la sostanza che si brucia, oppure ancora se dice ह̌k $\alpha \sigma \tau o \varsigma$ [ciascuno]

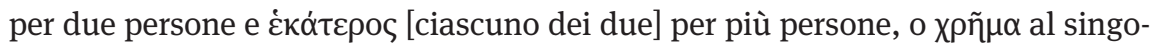

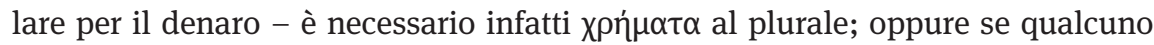

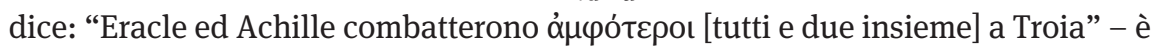

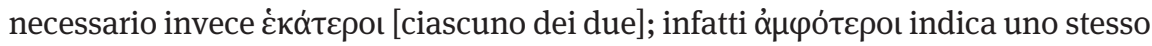

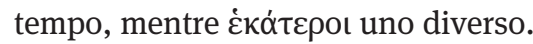




\section{Note}

$\mathbf{1}<\mathbf{0}$ ن̀: il testo tràdito è in contraddizione con la definizione di acirologia; l'integrazione restituisce un senso compiuto e coerente ed è facilmente giustificabile con un errore per aplografia. Inoltre, il nesso oủ kupíws è frequentemente utilizzato in testi grammaticali per indicare improprietà di linguaggio (cf. Harp. $\beta$ 2.2; Hermog. Id. 2.5.37; Ph. Legum allegoriarum libri 2.10.1; Phot. o 84.2).

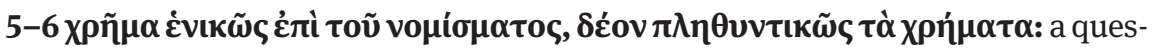
to esempio $\mathbf{N}$ (assieme ai suoi descripti $\mathbf{L}$ e $\mathbf{M}$ ) e $\mathbf{R}$ fanno seguire la precisazione del

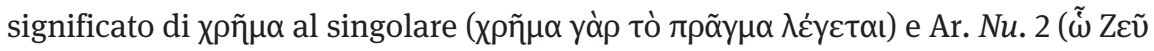
$\beta \alpha \sigma \iota \lambda \varepsilon \tilde{v}$ Tò $\chi \rho \tilde{\eta} \mu \alpha \tau \tilde{\omega} v v v \kappa \tau \tilde{\omega} v$ öбov). Il verso aristofaneo è citato nella Suida e negli scolii alle Nuvole come esempio di $\chi \rho \tilde{n} \mu \alpha$ al singolare quando tuttavia non vuol dire

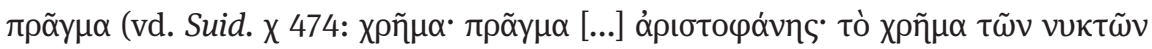

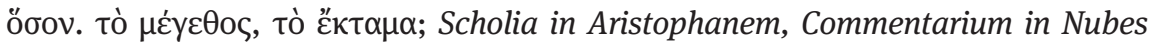

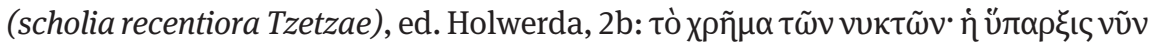

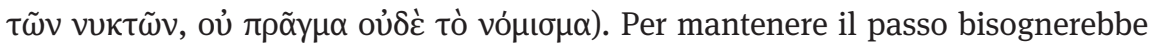
immaginare una lacuna dopo $\pi \rho \tilde{\alpha} y \mu \alpha$ da integrare con qualcosa come <ỉ tò

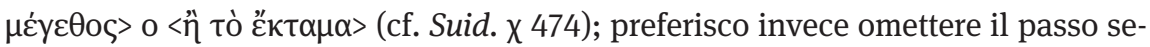
guendo $\mathbf{Q}$ e ritenendolo un'interpolazione o una glossa intrusiva di un copista che, sentendo l'esigenza di esplicitare il significato di $\chi \rho \tilde{\eta} \mu \alpha$ al singolare, ha maldestramente ridotto un qualche lemma lessicografico o scoliastico. Nel solo $\mathbf{R}$ troviamo

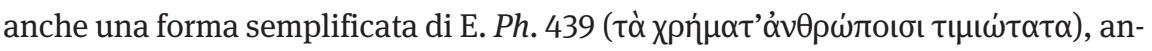
ch'essa da considerarsi un'interpolazione.

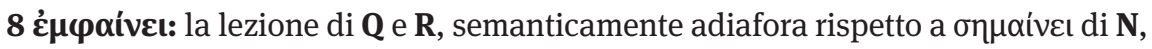
è da preferire in quanto a un tempo lectio antiquior e lectio difficilior. L'innovazione è probabilmente da attribuire a Konstantinos Laskaris, ossia il copista di $\mathbf{N}$, il quale frequentemente interveniva nei testi che copiava, soprattutto quelli paraletterari. ${ }^{13}$

Ringraziamenti: con piacere ringrazio Fabio Vendruscolo per l'aiuto e i consigli sul testo e sulla tradizione manoscritta.

13 Sicuramente sue sono ad esempio le innovazioni al testo di Palefato nel manoscritto Madrid, Biblioteca nacional de España, gr. 4628, per le quali vd. E. Villa, "Verso una nuova edizione critica di Palefato: la classe A”, in: M. Alganza Roldán, A. Zucker (cur.), Estudios sobre Palefato, Granada (in corso di pubblicazione). 